\title{
Impedimetrische und optische Detektion extrazellulärer DNA im Wundmodell
}

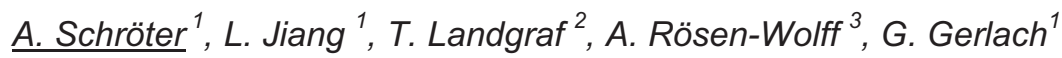 \\ ${ }^{1}$ Institut für Festkörperelektronik, TU Dresden, 01062 Dresden, Deutschland \\ anna.schroeter@tu-dresden.de \\ ${ }^{2}$ Fraunhofer-Institut für Verkehrs- und Infrastruktursysteme, Zeunerstr. 38 \\ 01069 Dresden, Deutschland \\ ${ }^{3}$ Klinische Forschung der Kinder- und Jugendklinik, Universitätsklinikum Carl Gustav Carus, \\ Fetscherstr. 74, 01307 Dresden, Deutschland
}

\begin{abstract}
Zusammenfassung:
Extrazelluläre DNA (ez-DNA) ist ein wesentlicher Bestandteil infektiöser Wundflüssigkeit. Die Erkennung einer Wundinfektion ist besonders bei der Behandlung chronischer Wunden vorteilhaft. Dafür wird ein impedimetrischer Sensor entwickelt, der ez-DNA detektiert. Die Performanz des Sensors kann durch ein mikroskopisches Nachweisverfahren ermittelt werden. Es wird ein fluoreszenzbasiertes Verfahren untersucht, das mit einer einfachen schwellwert-basierten Segmentierung bereits gute Übereinstimmungen mit der Impedanzmessung bringt. Im Zeitverlauf ändert sich die Fluoreszenzfläche tendenziell ähnlich der Impedanzänderung. Die Untersuchung höherwertiger Segmentierungsalgorithmen zeigt, dass eine Level-Set-Methode mit aktiver Konturerkennung ein geeignetes Verfahren ist, um ez-DNA zu segmentieren. Es wurden 84\% der DNA-Strukturen richtig erkannt bei einer Streuung von nur $3 \%$.
\end{abstract}

Stichworte: Wundmonitoring, Impedanzsensor, optische DNA-Erkennung, Fluoreszenzmikroskopie, Level-Set-Methode, Segmentierungsalgorithmus.

\section{Einleitung}

Chronischer Wunden sind schlecht heilende traumatische Schädigungen der Haut und der tieferen Gewebeschichten. Sie verursachen bei Betroffenen über Monate, teilweise über Jahre hinweg Schmerzen und eine Einschränkung der Mobilität des verletzten Körperteils. Zusätzlich müssen diese Patienten regelmäßig die Klinik aufsuchen und Verbandswechsel durchführen lassen. Dieser erfolgt häufig vorsorglich alle zwei bis drei Tage wegen möglicher Infektionen. Moderne hydroaktive Verbände weisen jedoch wesentlich längere Verweildauern auf, als in der Praxis umgesetzt werden [1]. Um diese Intervalle zu verlängern und den Stress für das Wundmilieu zu vermindern, ist der Einsatz eines Wundmonitorings sinnvoll.

\section{Sensor- und Validierungskonzept}

An der Technischen Universität Dresden wird an der Entwicklung eines Sensorkonzepts für diese Aufgabe geforscht [3]. Dazu wird ein Abwehrmechanismus genutzt, der mit einem Impedanz-basierten Verfahren erfasst wird. Im Falle einer massiven Besiedlung einer Wunde mit Pathogenen wird die Immunabwehr aktiviert und neutrophile Granulozyten in großer Anzahl rekrutiert. Diese reagieren besonders in Wunden nicht nur als Fresszellen (Phagozytose), sondern auch mit der Ausschleusung ihres DNA-Materials (Netose). Charakteristisch dafür ist die weißliche Verfärbung des Wundsekrets. Die ez-DNA ist in der Lage, viele Pathogene zu immobilisieren und mit bakteriziden Substanzen unschädlich zu machen [2]. Das massive Auftreten der DNA, auch Neutrophil Extracellular Traps, kurz NETs, genannt, führt zu einer charakteristischen Änderung im Impedanzspektrum. Im Wundmodell wurden bei einer charakteristischen Frequenz von $17,5 \mathrm{kHz} \mathrm{Im}$ pedanzänderungen von etwa $35 \%$ beobachtet [3].

Um einen Zusammenhang zwischen dem biologischen Ablauf der DNA-Ausschleusung und der Impedanzänderung herstellen zu können, ist es notwendig, etablierte Referenzmessverfahren einzubeziehen, beispielsweise die Fluoreszenzmikroskopie [4]. Die Herausforderung besteht darin, den Anforderungen beider Methoden, der optischen und der impedimetrischen, gerecht zu werden, um ein bio-elektrochemisches Modell für die DNAbedingte Impedanzänderung zu etablieren. 


\section{Methoden}

Das an der Universitätsklinikum Carl Gustav Carus entwickelte Wundmodell benutzt zur Simulation eines infektiösen Prozesses humane neutrophile Granulozyten von freiwilligen Spendern. Diese werden in mit Elektroden ausgestatteten Zellkulturschalen kultiviert. Eine PolyL-Lysin-Schicht erleichtert die Anhaftung der Zellen, die sie während der DNAAusschleusung bevorzugen. Die Stimulation zur Abwehrreaktion erfolgt über ein chemisches Stimulans, das Phorbol12-Myristat13-Azetat (PMA). Die Elektroden werden mit einem Adapter zweikanalig mit dem Impedanzspektrometer verschaltet. Das Messgerät erfasst die zeitliche Änderung des Impedanzspektrums.

In einem Vorexperiment wurde die Anwendbarkeit einer quasi-parallelen Fluoreszenzerfassung überprüft. Dazu wurde die Impedanzmessung zu einem bestimmten Zeitpunkt nach der Stimulation abgebrochen, die Proben mit einem Fluoreszenzfarbstoff angefärbt und mit Paraformaldehyd fixiert. Als Referenz sind stets unstimulierte Proben mitgeführt. Der Farbstoff Sytox Green durchdringt nicht die Zellmembran und färbt somit spezifisch ez-DNA an. Die Zellkulturschalen werden mit einem Fluoreszenzmikroskop gerasterte hochaufgelöste Aufnahmen erstellt (Ausschnitt: Bild 1). Das Resultat ist ein zusammengesetztes Fluoreszenzbild des gesamten Wells. In den Vorversuchen wurde die Fläche der fluoreszierenden Bildbereiche bestimmt. Bei gleichbleibender Gesamtfläche des gerasterten Wells hängt diese Fläche von der Anzahl der ez-DNA im Well ab [4]. Die Fläche wird mit einem festgelegten Intensitätsschwellwert ermittelt.

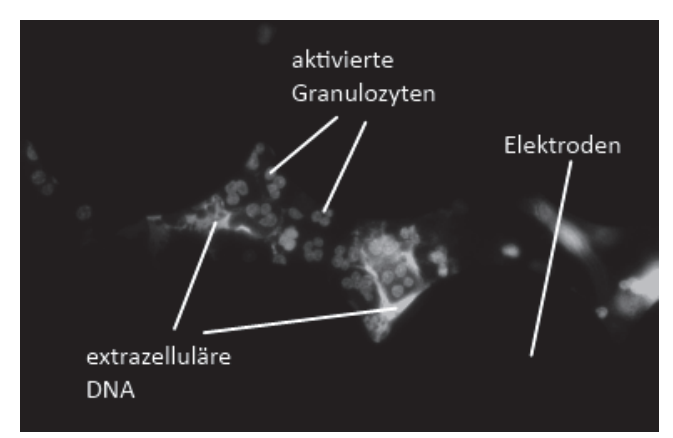

Bild 1. Stimulierte Granulozyten auf Elektroden (Abschattung) mit ihrer extrazellulären DNA.

Diese relativ grobe Schätzung lässt sich durch Bilderkennungsalgorithmen verbessern. Diese wurden in Matlab implementiert und an den aufgenommenen Bildern angewendet. Folgende Verfahren wurden für die Segmentierung betrachtet:

$>$ schwellwertbasierte Erkennung (SWB)

$>$ Wasserscheidentransformation (WST)

\section{$>$ Level-Set-Methode (LSM)}

Bei der schwellwert-basierten Erkennung werden Bildpunkte, die einen bestimmten Schwellwert überschritten haben, dem Vordergrund zugeordnet. Das in Matlab integrierte Verfahren mit automatischer Schwellwerterkennung benutzt das Verfahren nach Otsu [5]. Bei diesem Verfahren wird der Schwellwert so festgelegt, dass die Varianz innerhalb der segmentierten Bereiche minimiert und zwischen den Bereichen maximiert wird.

Die WST ist ein mathematisches Werkzeug, welches für die Bildverarbeitung entwickelt wurde. Eine plastische Veranschaulichung ist das Bild mit seinen Grauwerten als topografische Höhenkarte. Die WST füllt, beginnend mit den niedrigsten Bereichen, das topografische Gelände mit Wasser von unten auf. Dabei entstehen abgegrenzte Seen. Diese Begrenzungen entsprechen denen der segmentierten Bereiche. Das in Matlab implementierte Verfahren ist in Meyer et al. [6] beschrieben.

Grundidee des Level-Set (LS) basierten Ansatzes ist die Definition einer dreidimensionalen LS-Funktion $\Phi$ über der zweidimensionalen Bildfläche. Die eigentliche Segmentierung erfolgt durch das Festlegen eines Schwellwerts für $\Phi$, dieser kann graphisch als Level interpretiert werden. Für den hier verwendeten Ansatz Active Contours Without Edges [9] erfolgt eine iterative Anpassung der LS-Funktion

$\frac{\partial \Phi}{\partial t}=E(\Phi)|\nabla \Phi|$

mit dem Anpassungsterm

$E(\Phi)=E_{G / M}+v^{*} E_{C}$.

Der erste Term $E_{G / M}$ beschreibt die iterative Anpassung an den Bildinhalt. Dazu werden für die Segmentierung und den Hintergrund Mittelwert und Streuung der Intensität ermittelt. Für jeden Bildpunkt wird dann anhand der Intensität die Wahrscheinlichkeit (Normalverteilung) für die Zugehörigkeit zur Segmentierung bzw. zum Hintergrund bestimmt, was einen positiven bzw. negativen Wert für $E_{G / M}$ zur Folge hat. Der zweite Term $E_{C}$ basiert auf der Länge der Kontur und spiegelt die Glattheit der ermittelten Segmentierung wider. Über den Gewichtungsfaktor $v$ kann die Glattheit der Segmentierung gesteuert werden.

Zur Bewertung der Segmentierungen wurde die Erkennungswahrscheinlichkeit 


$$
P_{r}=\frac{r_{p}+r_{n}}{r_{p}+f_{p}+r_{n}+f_{n}}
$$

verwendet. Hier ist $r_{p / n}$ die Anzahl der richtig positiv/negativ segmentierten Bildpunkte und $f_{p / n}$ mit die Anzahl der falsch positiv/negativ segmentierten Bildpunkte. Als Vergleichsbasis dienen zehn manuell markierte Aufnahmen.

\section{Ergebnisse}

Die Bildaufnahme im Vorexperiment zeigte deutlich die Bildung von ez-DNA in den Wells (Bild 1). Allerdings konnte auch in unstimulierten Proben ez-DNA ausgemacht werden. Eine quantitative Auswertung ist in Bild 2 dargestellt. Vor der Stimulation zum Zeitpunkt 0 ist bereits ez-DNA vorhanden. Wir vermuten eine Vorstimulation durch die rauen Bedingungen für die Zellen während der Präparation. Diese ist unvermeidbar und wurde bereits in anderen Veröffentlichungen beschrieben [8]. Die unstimulierten Proben zeigen eine leicht verminderte Fluoreszenz, während die Impedanz unverändert ist. Die Minderung interpretieren wir als NETAbbau, verursacht durch DNAsen des verwendeten autologen Serums. Der Abfall ist ebenso in stimulierten Proben zu beobachten, die einen Höhepunkt beschreiben, der vermutlich überschritten wird, wenn alle möglichen Zellen rekrutiert wurden um DNA auszuschleusen, und nur noch der Abbauprozess dominiert. Die Fluoreszenzwerte ändern sich tendenziell ähnlich zur Impedanz. Lediglich um das Maximum herum sind starke Abweichungen zu beobachten, die davon abhängen, ob die Probe ihr Maximum überschritten hat. Einen weiteren Einfluss haben die ca. 20 Minuten, die zum Anfärben gebraucht werden. In dieser Zeit ändert die Probe ihren Zustand bereits kontinuierlich.

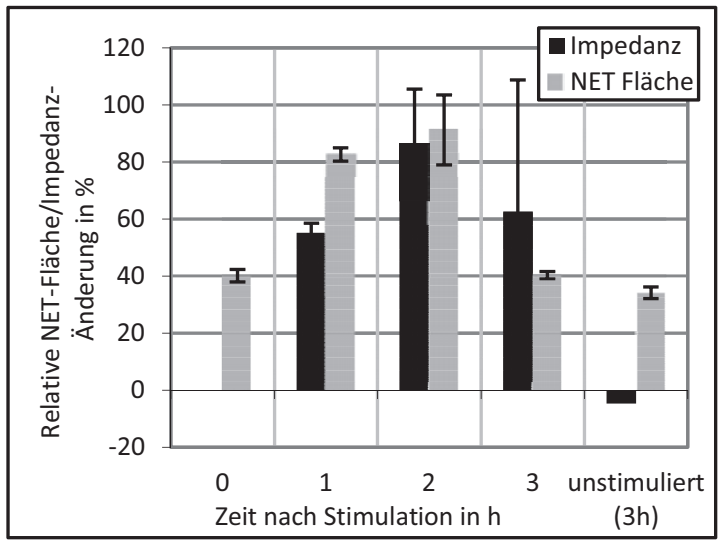

Bild 2. Verlauf der Impedanzänderung (bezogen auf den Anfangswert bei $17,5 \mathrm{kHz}$ ) und der relativen Fluoreszenzfläche (bezogen auf die maximale Fläche) in Abhängigkeit von der Stimulation.
Eine weitere Möglichkeit zur Verbesserung der mikroskopischen Auswertung ist die Anwendung der oben genannten Segmentierungsalgorithmen (Bild3). Prinzipiell zeigen alle drei Verfahren recht gute Erkennungswahrscheinlichkeiten, wobei mit WST die schlechtesten Ergebnisse auftraten. LSM zeigt eine deutlich geringere Streuung der Erkennungswahrscheinlichkeit. Da die LSM mehr Informationen einbezieht ist die höhere Zuverlässigkeit nachvollziehbar. Die LSM zeigt damit insgesamt das beste Ergebnis.

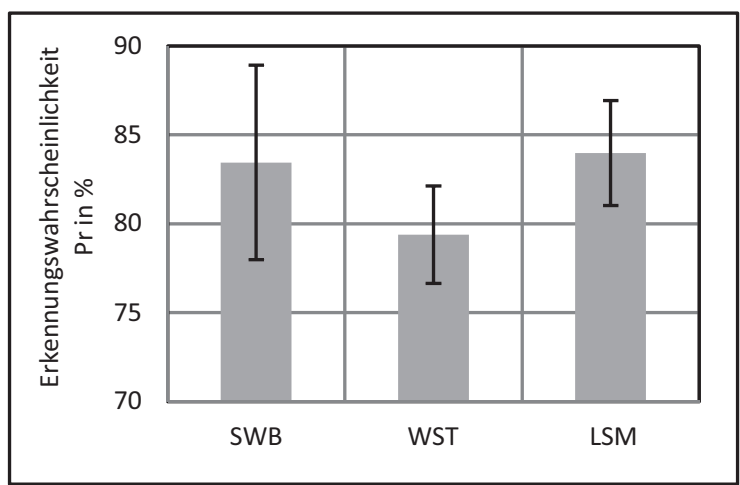

Bild 3. Erkennungswahrscheinlichkeit für die Segmentierung mit SWB, WST und LSM.

\section{Schlussfolgerungen}

Die mikroskopbasierte Erkennung der ez-DNA ist eine sinnvolle Ergänzung zur Impedanzspektroskopie. Das konnte in einem Vorexperiment gezeigt werden. Die anschließende Bildverarbeitung birgt viel Verbesserungspotenzial dem wir uns für die Segmentierung widmeten. Wir konnten zeigen, dass eine rechnerbasierte Erkennung mit passenden Algorithmen, wie die Level-Set-Methode, eine Quantifizierung der fluoreszierenden Fläche infolge von ez-DNA ermöglicht. Das Verfahren wählt im Vergleich zu einer manuellen Markierung die Fläche mit $84 \%$ Übereinstimmung aus benötigt aber nur einen Bruchteil des Zeitaufwands. Auf dieser Grundlage sollen im nächsten Schritt weiterer Vergleichsaufnahmen mit der LSM ausgewertet werden und mit den Ergebnissen der Impedanzspektroskopie verglichen werden. Außerdem ist geplant, durch die Weiterentwicklung der Klassifizierung, Stadien der DNAAusschleusung unterscheiden zu können. Damit sind bessere physikalische Rückschlüsse zur Wirkung der ez-DNA auf die Impedanz der Probe möglich, was eine bedeutende Weiterentwicklung des impedimetrischen Wundsensors bedeutet.

\section{Danksagung}

Das IGF-Vorhaben 17826 BR der Forschungsvereinigung Forschungskuratorium Textil e.V., Reinhardtstraße 12-14, 10117 Berlin wurde 
über die AiF im Rahmen des Programms zur Förderung der industriellen Gemeinschaftsforschung (IGF) vom Bundesministerium für Wirtschaft und Technologie aufgrund eines Beschlusses des Deutschen Bundestages gefördert.

\section{Literatur}

[1] Bundesverband Medizintechnologie e. V., Informationsbroschüre Wirtschaftlichkeit und Gesundheitspolitik: Einsatz von hydroaktiven Wundauflagen. Medlnform (2011).

[2] V. Brinkmann, U. Reichard, C. Goosmann; B. Fauler, Y. Uhlemann, D. S. Weiss, Y. Weinrauch, A. Zychlinsky, Science 5303 (5663), 1532-1535 (2004); doi: 10.1126/science. 1092385

[3] A. Schröter, A. Walther, K. Fritzsche, J. Kothe, A. Rösen-Wolff, G. Gerlach, Procedia Chemistry 6, 175-183 (2012); doi: 10.1016/j.proche.2012.10.144

[4] T. A. Fuchs, U. Abed, C. Goosmann, R. Hurwitz, I. Schulze, V. Wahn, Y. Weinrauch, V. Brinkmann, A. Zychlinsky, J. Cell Biol. 176 (2), 231-241(2007); doi: 10.1083/jcb.200606027

[5] N. Otsu, IEEE Trans. on Systems, Man, and Cybernetics 9 (1), 62-66 (1979);

[6] F. Meyer, Signal Processing 38 (1), 113-125 (1994); doi: 10.1016/0165-1684(94)90060-4

[7] T.F. Chan and L.A. Vese. Active contours without edges. IEEE Transactions on Image Processing 10 (2), 266-277, 2001; doi: 10.1109/83.902291;

[8] H. Parker, M. Dragunow, M.B. Hampton, A. J. Kettle, C. C. Winterbourn, Journal of Leukocyte Biology 92 (4), 841-849 (2012); doi: $10.1189 / \mathrm{jl} b .1211601$ 